\title{
Effect of a newspaper article on the response to a postal questionnaire
}

Postal questionnaires are often used for the collection of medical research data. Response may be modified by many factors, ${ }^{1}$ and incentives to achieve a positive response have been experimentally tested. ${ }^{12}$ Here we report on a trial which tested whether an accompanying newspaper article with a description of the study might influence the response to a postal questionnaire.

The children of 2637 women who participated in two randomised trials of routine ultrasonography in pregnancy ${ }^{3}$ have been followed up to study any harmful effects of screening. Addresses of 2390 women were traced by November 1,1988 , and they were sent a postal questionnaire with an informative letter and a postage paid envelope. The questionnaire contained 66 closed end questions, some specifically addressing the child's health (hearing, vision, handedness). Since 91 women $(3 \%)$ could not be traced by the post service, 2299 women are likely to have received the questionnaire.

To avoid unnecessary anxiety among pregnant women no information about the study was disclosed to the public. Nevertheless, the study received press coverage on day 24 after mailing, after which we observed a strong increase in the number of returned questionnaires.

We decided to test whether an accompanying newspaper article with a description of the study might influence the $716(31 \%)$ who had not returned the questionnaire after two months. A photocopy of the article was randomly allocated with a letter of reminder, and response was monitored for 30 days. The article was then forwarded to those who were randomised to the control group, and still had not responded.
The results were analysed with a Kaplan-Meier survival procedure, and equality of response probability between the groups was tested with $\log$ rank $\chi^{2}$ statistics. The shape of the response curve indicated that the hazards were proportional, and an overall odds ratio of response between groups was calculated.

The figure shows that women who did not receive a newspaper article describing the study were less likely to return the questionnaire (log rank $\chi^{2}=5.36, p=0.02$, odds ratio $=0.79,95^{\circ} \circ$ confidence interval $0.66-0.96$ ), and over three months following the 30 day trial their response never caught up with that of those who received the article with their letter of reminder (odds ratio $=0 \cdot 67,95^{\circ}$ confidence interval $\left.0.56-0 \cdot 80\right)$.

These results suggest that a postal questionnaire with an enclosed newspaper article describing the study may be more favourably responded to than a questionnaire without such an incentive.

KJELL Å SALVESEN LARS J VATTEN University Hospital 7006 Trondheim Norway

1 Dillman DA. Mail and telephone surveys. The total design method. New York: John Wiley, 1978

2 Cartwright A. Some experiments with factors that might affect the response of mothers to a postal questionnaire. Stat Med 1986; 5: 607-17.

3 Bakketeig LS, Eik-Nes SH, Jacobsen G, et al. Randomised controlled trial of ultrasonographic screening in pregnancy. Lancet 1984; ii: 1179-82.

4 Eik-Nes SH, Økland O, Aure JC, Ulstein M. Ultrasound screening in pregnancy: a randomised controlled trial. Lancet 1984; i: 1347.
Response to a reminder questionnaire among women with a newspaper article enclosed $(n=392)$ and without a newspaper article enclosed $(n=324)$

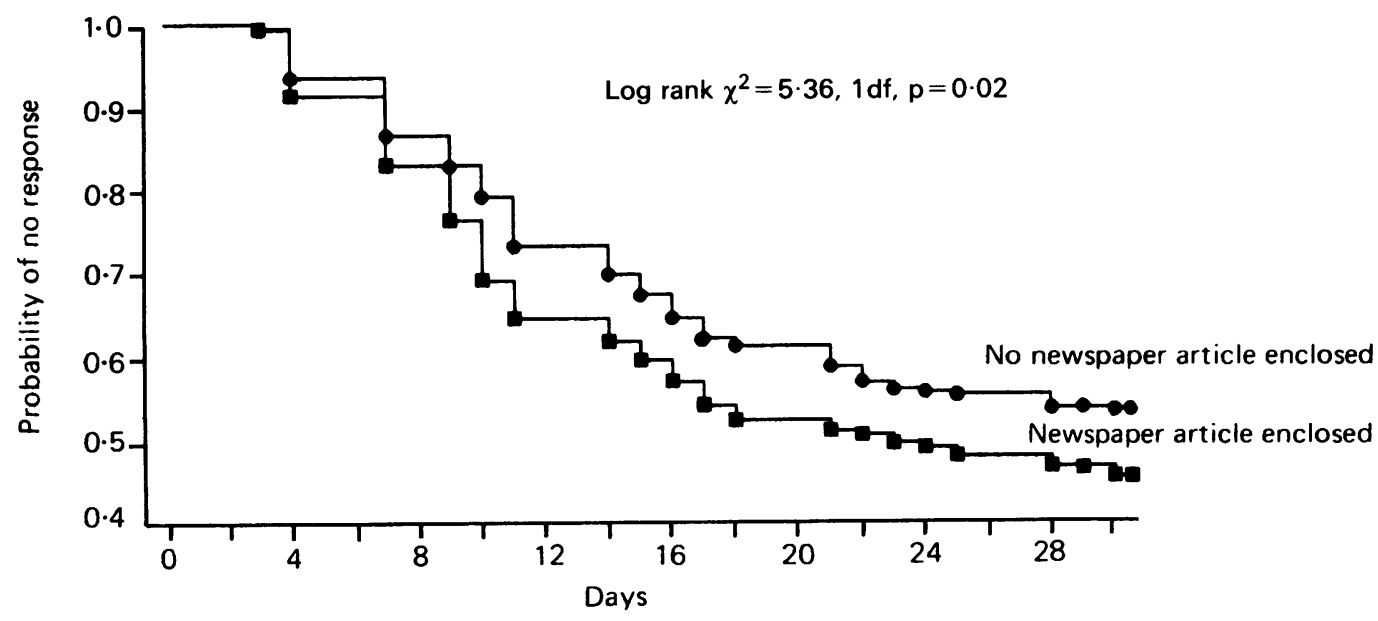

